\title{
Lorey, Isabell (2020): Demokratie im Präsens. Eine Theorie der politischen Gegenwart
}

\author{
Berlin: Suhrkamp. 217 Seiten. $20 €$
}

\section{Emanuel Richter}

Angenommen: 31. März 2021 / Online publiziert: 26. April 2021

(C) Der/die Autor(en) 2021

Isabell Lorey knüpft in ihrem Buch an verbreitete Gegenwartsdiagnosen an. Diese stellen in Bezug auf die Funktionsfähigkeit der Demokratie irritiert und ratlos fest, dass sich viele Menschen durch die politischen Organe und Entscheidungsprozesse nicht angemessen repräsentiert fühlen. Gruppen, die sich als Randständige oder Minderheiten definieren, finden weder ausreichend Gehör noch Stimme und hinter ihrer spezifischen Problemlage scheint der allgemeine Strukturdefekt einer Demokratie auf, die über ihre Formen der politischen Repräsentation systematisch die Diskriminierung bestimmter Bevölkerungskreise betreibt. Das Ziel der politischen Gleichheit wird so verfehlt. Lorey buchstabiert diesen Befund gendertheoretisch aus und verbindet ihn mit einer modelltheoretischen Betrachtung von politischen Protestbewegungen.

Ausgangspunkt aller Überlegungen muss naheliegenderweise die Frage sein, wer in der Demokratie zum Demos gehört und wie man in ihn hineingelangt. Lorey kritisiert die Verengung der liberalen Demokratie auf eine „,maskulinistische, bürgerliche und ausschließende Grundkonstitution“ (S. 8). Für Lorey folgt daraus die Ungleichheit der Geschlechter sowie, in einem allzu plakativ ausfallenden Blick auf die internationalen Gesellschaften, ein von Weißen dominierter, neoliberal grundierter Nationalismus. Ein patriarchalisch besetzter Fortschrittsglaube präge die Verständnisse von Geschichte und Gegenwart und zementiere die exkludierenden Repräsentationsstrukturen. Die Demokratie verkomme zu einer in Konventionen befangenen Elitenherrschaft. Dem setzt Lorey das „Präsentistische“ entgegen, eine Figur, die dem Fundus der radikalen Demokratietheorie entstammt. Dieses „Präsentistische“ sperrt sich gegenüber den patriarchalischen „Gegenwartsfetischismen“ (S. 17) und fordert eine immer wieder erneuerte Klärung dessen, wer zum ,wir“ des Demos gehört, aus-

E. Richter $(\bowtie)$

Institut für Politische Wissenschaft, RWTH Aachen, Aachen, Deutschland

E-Mail: richter@ipw.rwth-aachen.de 
geführt als ein protestförmiges Aufbegehren gegen die exkludierenden politischen Konventionen. Die Demokratie wird so zu einem Kampfplatz um die Sichtbarmachung von Ausgeschlossenen, ihre repräsentativen Funktionsmechanismen werden durch spontan und unerwartet auftretende Störmanöver permanent erschüttert. Vor dem Moment der Konstituierung eines Volkes lauert demnach die unbändige Kraftquelle einer „Multitude“, die jeglichen Impuls zur „Schließung“ der Demokratie in Bezug auf den Mitgliederkreis und auf ihre Funktionsweise zu torpedieren vermag.

Das sind auffällig komplizierte und zugespitzte Vorstellungen von den Erscheinungsformen gelungener Demokratie, die auf vielen modelltheoretischen Voraussetzungen und begrifflichen Vorverständnissen beruhen. Lorey widmet den größten Teil des Buches dementsprechend den Referenzen auf politische Theoretiker, die nach ihrer Überzeugung zur Klärung der präsentistischen Pointe beitragen. Rousseau kommt als Verfechter einer „Volkssouveränität“ zu Wort, die nicht von Repräsentationsvorstellungen kontaminiert ist und die Verbindung zur unbestimmbaren „Multitude“ bewahrt. Jacques Derrida wird mit seiner Sentenz von der ,démocratie à venir“ als Referenzautor für die Flüchtigkeit, Unbestimmbarkeit und Unwägbarkeit der Demokratie gehandelt. Walter Benjamin wird als Theoretiker der ,unzeitgemäßen“, die Vergangenheit unkonventionell, widerständig und emanzipatorisch aufnehmenden Gegenwart bemüht. Mit Michel Foucault vertieft Lorey die vorausgehenden Modellkomponenten und betont das Infinite, Unbegrenzte, Unabgeschlossene, Nichtlineare und Undeterminierte der Demokratie. Die unkalkulierbare, situative „Bewegung“ tritt in den Vordergrund. Mit Antonio Negri verdeutlicht Lorey schließlich ihre Vorstellung von der „Multitude“, die als Quelle für Inklusionsansprüche den Prozess der Konstituierung von Demokratie unnachgiebig offenhält und damit auch die patriarchalischen Verkrustungen aufbricht.

Damit leitet Lorey über zu einer Art Projektion ihrer Demokratievorstellung auf eine Reihe von protestförmigen politischen Bewegungen in der Gegenwart, die sie als Verkörperungen einer solchen „Demokratie im Präsens“ betrachtet. Die in diesen Bewegungen zum Ausdruck kommende Ablehnung von repräsentativen Mechanismen, das Absehen von definierten Zielvorgaben, der aufständische Charakter und nicht zuletzt die queere Vielfalt der Mitglieder wertet Lorey als völlig neuartige, im Sinne ihrer Modelltheorie vielversprechende Erscheinungsformen von Demokratie, die nicht nur innovative Subjektverständnisse hervorbringen, sondern eine ganz neuartige Praxis der Demokratie. Von einer „Projektion“ zu sprechen, erscheint mir deshalb gerechtfertigt, weil die von Lorey entfaltete Verbindung zwischen präsentistischer Demokratietheorie und politischen Bewegungen den emanzipatorisch engagierten Akteurinnen und Akteuren unterstellt, mit ihrem Protest auf der Straße nicht nur für politische Sichtbarwerdung, Anerkennung und politische Veränderungen zu kämpfen, sondern gleichzeitig gegen die Aporien in den Modellkonstruktionen von Demokratie aufzubegehren. Das Unkonventionelle und Widerständige ist aber wohl eher umgekehrt Ausdruck eines durch politische Demütigung und Empörung ausgelösten, eruptiven Aufstands gegen unzulängliche demokratische Praktiken, die den Demokratietheoretikern die Augen öffnen für die Inkonsistenzen des liberalen Demokratiemodells. Die Funktionslogik der Demokratie ist nicht deckungsgleich mit situativem Handeln, mit Anerkennungskämpfen minoritärer Bewegungen oder mit einer protestförmigen, genderbezogenen Identitätsverweigerung. Die „Multitu- 
de" kann sich nicht in ausgesuchten demokratischen Akteurinnen und Akteuren verkörpern, sondern sie kann ihre Überzeugungskraft nur als ein mit dem Aufstand mitlaufendes Erklärungsmodell entfalten, das die Diskrepanz zwischen einer wechselhaften Menge an Menschen, die nach Berücksichtigung in der politischen Sphäre streben, und dem geschlossenen, exklusiven Kreis eines konstituierten Volks aufarbeitet. Zudem weist die verbreitete Unzufriedenheit mit den herrschenden Demokratieformen eine wesentlich breitere Gestalt auf als nur den protestförmigen Rekurs zur Basisdemokratie. Allzu oft wird gerade umgekehrt die Verzerrung der repräsentativen Demokratie zu einer autoritären Elitenherrschaft als Antwort auf die Erschöpfungssymptome der Demokratie gepriesen.

Die „Demokratie im Präsens“ lässt sich daher nicht plausibel zum Gegenmodell gegen die liberale Demokratie stilisieren, und sie lässt sich damit auch nicht als umfassende Strategie zur Bewältigung von Krisen der Demokratie handeln. Sie entfaltet ihre Wirkung stattdessen als ein konzeptionelles Muster für die Ermittlung struktureller Defekte. Wenn man sich des unauflösbaren Spannungsverhältnisses zwischen Theorie und Praxis der Demokratie bewusst bleibt und die Protestbewegungen nicht zu Helden politischer Theoriebildung stilisiert, dann liest sich Loreys Buch mit Gewinn als eine höchst anregende Auseinandersetzung mit der Vielschichtigkeit demokratischer Gestaltungsprinzipien und Wirkungsmechanismen.

Funding Open Access funding enabled and organized by Projekt DEAL.

Open Access Dieser Artikel wird unter der Creative Commons Namensnennung 4.0 International Lizenz veröffentlicht, welche die Nutzung, Vervielfältigung, Bearbeitung, Verbreitung und Wiedergabe in jeglichem Medium und Format erlaubt, sofern Sie den/die ursprünglichen Autor(en) und die Quelle ordnungsgemäß nennen, einen Link zur Creative Commons Lizenz beifügen und angeben, ob Änderungen vorgenommen wurden.

Die in diesem Artikel enthaltenen Bilder und sonstiges Drittmaterial unterliegen ebenfalls der genannten Creative Commons Lizenz, sofern sich aus der Abbildungslegende nichts anderes ergibt. Sofern das betreffende Material nicht unter der genannten Creative Commons Lizenz steht und die betreffende Handlung nicht nach gesetzlichen Vorschriften erlaubt ist, ist für die oben aufgeführten Weiterverwendungen des Materials die Einwilligung des jeweiligen Rechteinhabers einzuholen.

Weitere Details zur Lizenz entnehmen Sie bitte der Lizenzinformation auf http://creativecommons.org/ licenses/by/4.0/deed.de. 